\title{
Strategic Management in the Emergency Department. Think Simple
}

Vassiliu P

The paper of Tsiftsis and colleagues [1], published in this issue is both novel and substantial. In the "dry" Hellenic environment the interest in scientific publishing diminished during the financial crisis, which relegated novel academic activities to a lower priority. The paper answers a focused and clinically applicable dilemma, with valid scientific methodology. The effort of the authors is most welcome under these circumstances, as the study was conducted by clinicians who deal with emergency cases, and who, over and above their clinician responsibilities, found the time to cooperate on the design and execution of an academic product.

Focusing on the field of trauma, they have elected to evaluate the efficacy of a diagnostic tool, ultrasonography $(\mathrm{U} / \mathrm{S})$, that needs no consumables, is noninvasive, does not convey radiation, can be used in situ by the clinician and can give substantial information (if not always a diagnosis) that allows the emergency physician to make rapid and objectively justified clinical decisions. In the Hellenic environment, all these features constitute a major medicoeconomic advantage.

We are all aware that all tertiary hospitals on emergency duty accept patients from the National Ambulance Services, as well as direct from the community, with no limitations. The volume and diversity of these cases is enormous, and not all comprise emergencies. Triage is conducted by capable doctors who identify the emergency, urgent, chronic and simple cases [2]. The system does not allow for the less urgent cases to be to deferred until the next day, as such a hospital, in the mind of the health consumers is "the end of the road". An answer and a definitive one, must be given to their problem, "here and now". On top of that, every case examined by the on-call doctors, no matter how simple, is evaluated exhaustively with paraclinical examinations, as medicolegal issues may be raised if a prestigious referral hospital "loses" a case. In a related study [2] on a tertiary hospital, even the simplest cases that presented at the surgical emergency room (ER), underwent complete blood and radiological evaluation. As these simple cases constitute the vast majority of the patients examined during the on-call period, the burden on the health system in terms of time and funds is easily recognized.

The paper of Tsiftsis and colleagues offers an argument to the emergency clinician for clearing safely, rapidly and at low cost the major volume of some of the simplest cases. The only investment that the system would need is have available in the surgical ER ultrasonograms and clinicians trained in their use.

The benefit of this paper reaches much further than its academic reward to the authors. The application of its recommendations, if expanded to other fields in addition to trauma, would have substantial beneficial impact on the functioning and costs of our emergency health system.

\section{References}

1. Tsiftsis D, El-Fellah N, Xatzivasilliou A, et al. The Value of Complete Ultrasonography Diagnosis in the Management of Selected Patients with Abdominal Trauma. Hellenic J Surg 2018;90:115-20.

2. Koumpoura A, Tsombanellis A, Vassiliu P. Evaluation of the surgical emergency cases on a Tertiary Hospital. Oral Presentation at the $24^{\text {th }}$ Scientific Symposium of the Scientific Society of Greek Medical Students. Athens April 27, 2018.

«Attikon» University Hospital, National and Kapodistrian University of Athens, Greece

Corresponding author: Vassiliu P, MD, PhD, FACS, FASCRS «Attikon» University Hospital, 1 Rimini St., 12462 Haidari, Athens, Greece, Tel.: +30 2105831 385, e-mail: pant.greek@gmail.com 